\title{
Introduction. Frontières et logiques de passage : l'ordinaire des transgressions
}

Introduction. Borders and the logics of crossing: everyday transgressions Introducción: Fronteras y lógicas de pasaje: la normalidad de la transgresiones

\section{Karine Bennafla et Michel Peraldi}

\section{(2) OpenEdition \\ Journals}

\section{Édition électronique}

URL : http://journals.openedition.org/conflits/17383

DOI : $10.4000 /$ conflits. 17383

ISSN : $1777-5345$

Éditeur :

CCLS - Centre d'études sur les conflits lilberté et sécurité, L'Harmattan

Édition imprimée

Date de publication : 20 décembre 2008

Pagination : 7-12

ISBN : 1157-966X

ISSN : 1157-996X

Référence électronique

Karine Bennafla et Michel Peraldi, « Introduction. Frontières et logiques de passage : I'ordinaire des transgressions », Cultures \& Conflits [En ligne], 72 I hiver 2008, mis en ligne le 19 mai 2009, consulté le 30 mars 2021. URL : http://journals.openedition.org/conflits/17383 ; DOI : https://doi.org/10.4000/ conflits. 17383 


\section{Introduction. Frontières et logiques de passage : I'ordinaire des transgressions}

\section{Karine BENNAFLA}

Karine Bennafla est géographe, maître de conférences à l'IEP de Lyon, actuellement en délégation CNRS au Centre Jacques Berque à Rabat. Elle travaille sur les dynamiques de changement et l'intégration territoriale des zones frontalières, avec des cas d'étude en Afrique subsaharienne et dans le monde arabe. A récemment publié : Bennafla K., El Karoui D., Sanmartin O., Géopolitique du Maghreb et du Moyen-Orient, Paris, Sedes, 2007.

\section{Michel PERALDI}

Michel Peraldi est anthropologue, directeur de recherche au CNRS, directeur $d u$ Centre Jacques Berque pour le développement des sciences sociales à Rabat. Il travaille sur les dynamiques migratoires dans le bassin méditerranéen, les circuits commerciaux informels entre Maghreb et Europe. A récemment publié : Peraldi M., Kokoreff M., Weinberger M. (dirs.), Economies criminelles et mondes urbains, Paris, PUF, 2007.

Tssus d'un colloque organisé à Tanger 1 , les articles de ce numéro ouvrent des Lerspectives géographiques variées pour décrire la modernité de mondes sociaux construits autour de lieux frontaliers, en tirant parti au niveau économique, culturel et politique de la rupture de souveraineté instituée par la frontière. Ce n'est certes pas nouveau de considérer que les frontières font monde et que s'organisent, en ces bords d'Etat, des lieux très paradoxaux, puisqu'ils semblent nier la possibilité même de la frontière, formant pont et continuité là où, souvent, le politique voudrait rupture et limite, socialement stériles, de l'exercice d'une souveraineté. De nombreux travaux ${ }^{2}$ ont déjà mis en évidence

1. Colloque international "Vivre et tracer les frontières dans les mondes contemporains ", Tanger, 31 janvier-2 février 2008, organisé par le Centre Jacques Berque de recherches en sciences humaines et sociales (Rabat).

2 . Bromberger C., Morel A. (dirs.), Limites flones, frontières vives, Paris, Editions des sciences de l'Homme, 2000; Hannerz U., " Frontières ", Revue internationale des sciences sociales, ${ }^{\circ} 154$, 1997, pp. 596-609; Vila P., Ethnography at the Border, Minneapolis / London, Cultural 
les divers usages de la frontière et évoqué la vie sociale, économique, culturelle qui continue bien au-delà des limites territoriales et politiques de la souveraineté, avec son lot d'hybridations et d'enchevêtrement créatifs. Dans la forme impériale de l'Etat, du reste, ce type de frontière floue et poreuse a été codifié et souvent parfaitement organisé comme, par exemple, le limes romain. Les articles présentés ici rappellent fort opportunément que les frontières sont des lieux avant d'être des lignes, et que l'existence même de ruptures en matière de souveraineté est facteur, sinon de solidarités affranchies ou remobilisées par cette rupture, du moins d'opportunités économiques et sociales.

L'intérêt de cette lecture anthropologique de la frontière tient au fait qu'elle tend aujourd'hui à être obscurcie par l'évolution des dispositifs de pouvoirs et la réorganisation des «tactiques » frontalières depuis la chute du mur de Berlin. Il apparaît que cet événement a été moins le dernier avatar de la logique des blocs qu'une préfiguration de notre modernité. Notre époque se singularise en effet par des dispositifs frontaliers dotés de mécanismes de contrôle et de surveillance de plus en plus élaborés ${ }^{3}$, et par l'édification de barrières physiques, murs et enceintes, qui visent l'étanchéité absolue de la séparation. Les murs de béton dressés entre Mexique et Etats-Unis (depuis 1993) ${ }^{4}$, entre Cisjordanie et Israël (depuis 2002) ${ }^{5}$ ou les enceintes de barbelés ourlant les enclaves espagnoles de Ceuta et Melilla sont les exemples les plus connus de ces dispositifs frontaliers qui prétendent filtrer, contrôler les flux (avec, en ligne de mire, les migrants clandestins) ou bien sécuriser et défendre contre des agressions externes. Telle la frontière israélo-égyptienne évoquée par Lisa Anteby-Yemini, un nombre croissant de limites territoriales connaissent depuis les années 1990 une mutation de leur rôle stratégique, la fonction militaro-défensive initiale étant supplantée par une mission de surveillance des migrations clandestines, surtout avec l'externalisation des politiques de contrôle frontalier entreprise par les pays du Nord. Ces systèmes de clôtures matérielles et de barrières juridico-administratives incarnent des fronts " passifs » où le rôle de l'armée est restreint à celui de police et de vigile et ils ne se rencontrent pas qu'aux frontières des territoires d'Etat : à l'échelle urbaine, les gated communities ${ }^{6}$ des quartiers résidentiels concrétisent pareillement désir d'entre-soi, fantasmes de peurs et crispations identitaires ${ }^{7}$ face à l'ouverture

Studies of the Americas / University of Minnesota Press, n¹3, 2003, pp. xi-xxxv ; Miller T., Sur la frontière, Arles, Actes Sud, 1992.

3. Voir Bigo D. (dir.), «Circulation et archipels de l'exception », Cultures $\mathcal{E}$ Conflits, n68, hiver 2007.

4. Travaux du COLEF (El Colegio de la Frontera Norte), Tijuana, Mexique.

5. Warshawski M., Sur la frontière, Paris, Stock, 2002.

6. Lakely E.J., Snyder M.G., Fortress America, Gated Communities in the United States, Washington DC / Cambridge, Brookings Institution Press / Lincoln Institute of Land Policy, 1997 ; Davis M., City of Quartz, Excavating the Future of Los Angeles, London, Verso, 1990 (éd. française : La Découverte, Paris, 1997); Le Goix R., "Les “communautés fermées" dans les villes des Etats-Unis. Aspects géographiques d'une sécession urbaine ", L'Espace géographique, 2001, vol.30, $\mathrm{n}^{\circ} 1$, pp. 81-93.

7. Bayart J.-F., L'Illusion identitaire, Paris, Fayard, 1996. 
généralisée du monde, à l'interdépendance accrue des espaces et au creusement des inégalités socio-économiques. Comme il sera vu dans le prochain numéro de Cultures $\mathcal{E}$ Conflits, également issu des rencontres de Tanger et consacré aux logiques de marquage, les murs actuels se caractérisent par une architecture de l'étanchéité, un déploiement de dispositifs de contrôle parallèles et une volonté de marquage, non seulement de limites de souveraineté, mais aussi de principes d'identité voire, comme le souligne P. Andreas, d'une " mise en scène identitaire 8 ». Dès lors, il apparait une disjonction de plus en plus radicale entre, d'une part, la logique de marquage identitaire à l'œuvre dans le déploiement des mises en scène et des dispositifs de contrôle aux frontières et, d'autre part, les continuités sociales, les opportunités économiques que ces liens sociaux organisent et dynamisent. Certaines frontières devenues hautement symboliques de politiques de cloisonnement, comme la frontière EtatsUnis - Mexique, Espagne - Maroc et, bien sûr, la frontière israélo-palestinienne, atteignent les sommets du paradoxe de par leur très haut degré d'intégration économique et le paroxysme des marquages identitaires manifestés.

Aussi spectaculaire soit-elle, l'édification de murs n'interrompt pas les mobilités et elle est même, en soi, comme tout défi, un appel à transgression, ce que suggère bien l'expression française « faire le mur », qui signifie à la fois le construire et s'en affranchir. De fait, en dépit du durcissement législatif et administratif des contrôles, malgré les velléités de bouclage ou de filtrage aux frontières, jamais les flux d'hommes, d'idées, de marchandises, de capitaux n'ont été aussi intenses et banalisés qu'aujourd'hui. Les progrès technologiques dans le domaine des transports et télécommunications, la fin de la Guerre froide avec la faillite de la plupart des régimes communistes à économie fermée, la domination d'une doxa néolibérale vantant les mérites de l'échange et de l'ouverture ainsi que les délocalisations accrues d'entreprises ont contribué à fluidifier les déplacements et ériger la circulation en norme. Célèbre pour son mur, la frontière américano-mexicaine enregistre annuellement plus de 400 millions de passages. Autre révélateur de l'ampleur des mobilités : les déplacements de touristes internationaux qui croissent depuis plusieurs décennies, au point d'atteindre près de 900 millions d'arrivées en 2007, selon l'Organisation mondiale du tourisme. Visibles ou invisibles, matériels et immatériels, ces divers flux empruntent des canaux tantôt institutionnels, tantôt «informels » ou illégaux même si, dans les faits, beaucoup suivent un mode de cheminement hybride et composite 9 . Bien entendu, la fluidité à travers les mailles politico-administratives fluctue selon les biens et les individus.

8. Andreas P., Border Games. Policing the US-Mexico Divide, Ithaca/London, Cornell University Press; Zureik E., Salter M. (eds.), Global Surveillance and Policing: Borders, Security, Identity, Cullompton, Willan Publishing, 2005 ; Willoughby R., "Immigration, race et sécurité à la frontière mexicano-californienne », Cultures E Conflits, n²6-27, 2001.

9. Voir Hibou B., La Privatisation des Etats, Paris, Karthala, 1999 ; voir l'analyse du fonctionnement du port de Naples de Saviano R., Gomorra. Dans l'empire de la Camorra, Paris Gallimard, 2007. 
La nationalité, les revenus, le bagage culturel, le « capital relationnel 10 » sont des paramètres déterminants pour circuler et jouer des frontières.

En montrant le hiatus entre l'attitude des Etats nigérian et camerounais engagés dans un conflit autour d'une île du lac Tchad et la coopération transfrontalière opérée "par le bas » par les populations riveraines qui se partagent les ressources, l'article d'Halirou Abdouraman souligne l'ambivalence et les contradictions de la frontière : être à la fois un obstacle et un adjuvant aux relations, ces effets se modulant selon les époques, les types d'acteurs et l'échelle spatiale considérée. Les différentiels créés ou scellés par la frontière stimulent les échanges et toutes sortes de mobilités - travailleurs, touristes, réfugiés, etc. - en particulier pour ceux qui savent jouer des décalages, faire preuve d'audace ou prendre des risques dans la perspective d'améliorer leur sort, de s'enrichir ou de vivre autrement. Pour les candidats migrants, originaires des pays du Sud, la traversée de la frontière est une épreuve souvent violente et traumatisante avec une issue bien incertaine, comme le rappellent les contributions de Sabine Guez, Lisa Anteby-Yemini et Dominique Vidal. Pour beaucoup en effet, la frontière est le lieu de renoncements et d'échecs scandés par des drames familiaux, un retour plus ou moins forcé vers le pays de départ, l'emprisonnement ou la mort. Plus symboliques mais aussi périlleuses et hardies que les aventures migratoires, les unions matrimoniales mixtes incarnent une forme de passage et de réinvention de la frontière selon «un continuum entre transgression et normalité » bien décrit par Daniel Meier à propos des mariages libano-palestiniens. Dans tous les domaines, les entraves instaurées par la frontière génèrent des stratégies de contournement et de résistance, poussent à l'inventivité et agissent au niveau commercial et économique comme un véritable aiguillon.

Que ce soit en Amérique centrale, en Afrique ou en Europe, les espaces frontaliers se révèlent des zones d'échanges et de passage intenses, à la faveur de collectifs d'appartenance (re)mobilisés, ou dans la continuité de pratiques et de savoir-circuler anciens : qu'on songe aux tribus bédouines du Neguev ou du Sahara reconverties à l'ère moderne en contrebandiers ou passeurs d'êtres humains, ou encore aux migrations pendulaires de travail décrites par Nagy Raluca dans la région montagneuse du Maramures (Nord de la Roumanie). Intensifiées depuis la chute des régimes communistes à partir de 1989, celles-ci s’inscrivent dans une tradition de mobilité déployée par des paysans contraints à la pluriactivité.

La formation d'espaces métropolitains continus chevauchant la frontière américano-mexicaine, à l'image de Ciudad Juarez - El Paso ou de San Diego Tijuana, est révélatrice de la perméabilité frontalière ${ }^{11}$. Ces doublons urbains dessinent des conurbations traversées par la frontière qui semble ici souder, plutôt que séparer, des villes distinctes. Ce principe de continuum urbain est

10. Grégoire E., Labazée E., Grands commerçants d'Afrique de l'Ouest. Logiques et pratiques d'un groupe d'hommes d'affaires contemporains, Paris, Karthala, 1993.

11. Gondard P., Revel-Mouroz J., La Frontière Mexique - Etats-Unis, IHEAL, 2001. 
bien près de se produire aux portes des enclaves espagnoles de Ceuta et Melilla au Maroc, malgré l'étanchéité des dispositifs frontaliers mis en place. Sur les marches, se développent des commerces, du trafic et des va-et-vient multiples, avivés par les écarts de richesse, les complémentarités socio-économiques, parfois la prohibition ${ }^{12}$. En position d'interface, les marchés frontaliers prospèrent, approvisionnés par des places marchandes intérieures plus ou moins distantes, selon une géographie mouvante qui réactive quelquefois de vieilles routes commerciales. Certains marchés se situent entre des territoires d'Etats en guerre et des populations entières, poussées aux périphéries par la fragilisation de leurs conditions de vie, se livrent à des contrebandes dont l'inventaire est encore loin d'être exhaustif. Avec les économies frontalières, fleurissent des collaborations et des échanges culturels et sociaux dans leur grande majorité placés sous le signe de l'informalité, de la débrouille, voire de la clandestinité. En retraçant des trajectoires individuelles de passeurs, Sabine Guez livre une fine description du narco-trafic à la frontière américano-mexicaine et souligne la complexité des liens entre narcotrafiquants, représentants du pouvoir politique et employés des agences gouvernementales. Le cas de cette frontière démontre bien, comme ailleurs au Proche-Orient ${ }^{13}$, que les machines de contrôle destinées à réguler et maîtriser la frontière, alimentent une recrudescence de violences, de trafics et de criminalités, en écho au perfectionnement croissant des instruments utilisés.

Ces trafics multiples ne doivent pas faire oublier que le différentiel frontalier est aussi le support de nouvelles mobilités professionnelles et résidentielles (à l'image des transhumances saisonnières du « troisième âge doré » européen vers le Maroc ou la Tunisie) et une ressource économique pour des pans entiers de l'industrie qui se sont transportés vers les frontières. Les usines maquiladoras à la frontière mexicaine, les délocalisations industrielles plus récentes des entreprises européennes vers les zones franches marocaines ou tunisiennes, puis roumaines ou polonaises, sont un facteur majeur de transformation des espaces frontaliers, sans pour autant gager d'un réel développement, c'est-àdire d'une amélioration générale du bien-être des populations locales. De son côté, le renforcement des dispositifs de garde frontaliers alimente une " économie de la sécurité » qui fait les beaux jours des sociétés privées de surveillance, des fabricants de matériel de détection et contrôle (caméras, capteurs, etc.) et des entreprises en bâtiment chargées de construire les murs.

Au final, en dépit des tentatives de mise en ordre, paradoxe et confusion règnent aux frontières, avec le caractère trouble des trafics et des acteurs engagés dans un jeu d'alliances ou de collaborations surprenantes, fréquemment ancrées sur des réseaux de relations personnelles. Il est à remarquer que la plu-

12 . Bredeloup S., Pliez O. (dirs.), « Migrations entre les deux rives du Sahara », Autrepart, n ${ }^{\circ 36}$, 2005 ; Bensaad A. (dir.), "Marges et mondialisation: les migrations transsahariennes ", Maghreb-Machrek, ${ }^{\circ} 185$, automne 2005.

13. Parizot C., «En attendant le Mur: Gestions israéliennes des mobilités palestiniennes pendant la seconde Intifada (2000-2006) », Migrations société, vol. 18, n¹07, pp. 15-39. 
part de ces sociétés transfrontalières modernes jouissent, dans les ensembles nationaux où elles sont incluses, d'un statut ambigu entre tolérance dévolue aux marginalités utiles et violence symbolique stigmatisante. En Afrique du Sud, la disparition des frontières raciales de l'apartheid en 1994 a été relayée par l'affirmation d'autres frontières intérieures (mentales et juridiques) entre d'une part, les Noirs sud-africains, fiers d'avoir recouvré une citoyenneté dont ils avaient été dépossédés et, de l'autre, des migrants africains venus d'autres pays du continent comme le Mozambique (D. Vidal). L'ambiguité caractérise également les politiques de frontières qui oscillent entre mise en scène militaire et encadrement touristique annonciateur de réconciliation (Corée du Sud), ou bien entre obsession sécuritaire et conduite humanitaire : les pratiques contradictoires de l'Etat israélien vis-à-vis des communautés de migrants forcés africains (L. Anteby-Yemini) sont éloquentes en la matière. Enfin, le flou prévaut à propos du statut des brûleurs et autres passeurs de frontières dont un nombre croissant ne rentre plus dans aucune catégorie juridique, en particulier quand il s'agit d'enfants nés et ayant vécu en transit, sur un mode itinérant. Ces situations complexes, brouillonnes et chaotiques observées à la frontière font de celle-ci un lieu d'avant-garde où s'opèrent, dans le bricolage, des innovations. 